\title{
Age-related Mitochondrial DNA Deletions Produce Fusion Peptides in Mice
}

\author{
Md. Ayub Ryhan, Md. Miraj Kobad Chowdhury*
}

Department of Genetic Engineering and Biotechnology, University of Dhaka, BANGLADESH

*Corresponding Contact:

Email:miraj@du.ac.bd

\begin{abstract}
Deletions in mitochondrial DNA occur from oxidative stress generated during physiological processes. Such deletions have been associated with ageing, cancer, and neurodegeneration. In this study, the outcome of mtDNA deletions has been investigated to justify the physiological effect of such mtDNA deletion. For this, five established and two predicted age-related murine mtDNA deletions were analyzed for fusion peptide. Three out of the five established deletions and all the predicted deletions produced fusion proteins like COXIIIND5, ND3-ND5, ATP6-ND5, and ND5-CYTB. Interestingly, we have observed that the partners of these fusion proteins are from different complexes. Thereby, these fusion proteins can produce chimeric complexes that could bypass the usual electron transport system and could make short-circuit across the mitochondrial membrane. Such event can provoke more oxidative stress leading to further accumulation of mtDNA damage and apoptosis.
\end{abstract}

Key words

Mitochondria, mtDNA deletion, Fusion peptide, Oxidative stress

\section{INTRODUCTION}

The genome contains all the genetic instruction necessary for the development, function, and reproduction of life (Boesch et al., 2011). Typically a mammalian cell possesses two sets of genomes; the nuclear genome (nDNA) and the mitochondrial genome (mtDNA) (Druzhyna et al., 2008). Mammalian mtDNA is about a $16 \mathrm{~kb}$ closed-circular dsDNA existed as several copies in the matrix of each mitochondrion. Mitochondria have evolved from purple non-sulfur bacteria according to endosymbiotic theory (Croteau et al., 1999). During this evolutionary process, mtDNA has been considered to undergo several deletions, which were integrated into the nuclear genome of the host cell (Jeppesen et al., 2011). Still, mtDNA undergoes some common deletions during increased oxidative stress from intracellular metabolism or from exogenous sources (Tann et al., 2011). However, the fate of the deleted 
fraction from mtDNA is still unknown. The major function of mitochondria is to produce energy in the form of ATP from food through aerobic respiration. To carry out the oxidative phosphorylation reaction (OXPHOS), mitochondrion needs five enzymatic complexes (named as complexes I, II, III, IV, and V) found in the inner membrane of mitochondria. The oxidations of carbohydrates and fatty acids produce NADH and FADH2 by Krebs cycle and $\beta$-oxidation cycle respectively. Electrons from NADH and FADH2 passed down to a series of donor and electronegative acceptor for the final reduction of oxygen to water. Energy found from each step of the reaction is used to pump protons from the mitochondrial matrix into the inter-membrane space. As mitochondria play the vital role in producing energy, cell signaling, cell differentiation and cell death, subsequent loss of function of mitochondrial DNA causing mitochondrial dysfunction is potentially harmful to the cell (Boesch et al., 2011). Such mitochondrial dysfunction has been reported to contribute to aging, neurodegeneration, cancer, type II diabetes, and some genetic disorders (Jeppesen et al., 2011). Though considered as a continuous evolutionary process, it is still unknown whether the deleted part of mtDNA still exists inside the mitochondrial matrix, or it finds a way to be incorporated into the nuclear genome, or it is degraded. Up to now, no research has been done to solve this issue. Interestingly, the answer to this question might open a new dimension in organelle biology, especially in the area of mitochondrial biology and nuclear transgenesis. In this research, the outcome of age-related mtDNA deletion in mice was studied. We have observed that such deletions produce fusion peptides which may contribute to the generation of short-circuit across mitochondrial electron transport chain.

\section{MATERIALS AND Methods}

1. Retrieve sequence of mitochondrial DNA of the mouse: The mitochondrial DNA (mtDNA) sequences used in this study were obtained from the National Centre for Biotechnology Information (NCBI) database.

2. Find out the position of deletion: Deletions with their associated direct repeats were identified in the genome sequence (Tanhauser et al., 1995) (Table 1).

Table 1: Direct repeats and their deletions in mouse mtDNA (Tanhauser et al., 1995)

\begin{tabular}{|c|c|c|c|}
\hline DNA repeat & Sequence & Position & $\begin{array}{l}\text { Deletion } \\
\text { size(bp) }\end{array}$ \\
\hline $\mathrm{D}-1$ & AGCCCTACTAATTAC & $\begin{array}{l}9,089-9,103 \\
12,956-12,970\end{array}$ & 3867 \\
\hline $\mathrm{D}-2$ & TCTTTGCAGGATT & $\begin{array}{l}8,844-8,896 \\
13,120-13,132\end{array}$ & 4326 \\
\hline D-3 & AAGCAAATCCATAT & $\begin{array}{l}9,553-9,566 \\
13,279-13,292\end{array}$ & 3726 \\
\hline D-13 & $\begin{array}{l}\text { CCCTCCTTCTAACAT } \\
\text { CCСТCCTTCCAACAT }\end{array}$ & $\begin{array}{l}8,677-8,691 \\
13,651-13,665\end{array}$ & 4974 \\
\hline D-14 & $\begin{array}{l}\text { CAATAATAGGATTCCCAATCG } \\
\text { CAGTATTAGGATTCCTAATCG }\end{array}$ & $\begin{array}{l}7,964-7,984 \\
13,216-13,236\end{array}$ & 5252 \\
\hline D-16 & $\begin{array}{l}\text { TACCCCTATTAATATTTTCC } \\
\text { TAACCCTAGTATTATTTTCC }\end{array}$ & $\begin{array}{l}11,881-11,901 \\
14,857-14,877\end{array}$ & 2976 \\
\hline D-17 & $\begin{array}{l}\text { ACTAATCCTAGCCCTAGCCC } \\
\text { AATAACCCTACCCCTAGCCC }\end{array}$ & $\begin{array}{l}1,094-1,113 \\
4,915-4,934\end{array}$ & 3821 \\
\hline
\end{tabular}

3. Determination of the resulting transcript and fusion protein after deletion: The nucleotide sequence after deletion only with the first direct repeat was determined. $1 \mathrm{~kb}$ from the 
upstream region and $1 \mathrm{~kb}$ from the downstream region (varies depending on the size of the deletion) were specified for each deletion. Open reading frames were identified at http://web.expasy.org/translate/ until any stop codon is found. For any exact direct repeat, only a single transcript or protein product was accounted.

4. Predicting the structure of the fusion protein: To predict three-dimensional structures, $\mathrm{CPH}$ models 3.2 (a web-based tool from http://www.cbs.dtu.dk) was used. Nucleotide sequences were uploaded to this server with FASTA format and the outlined threedimensional structures were predicted by PDB3D model (high-speed 3D molecule rendering applet). Jmol software was also used to predict three-dimensional structures for its capability to read multiple file types including PDB, CIF, SDF, MOL, PyMOL PSE files, and Spartan files.

\section{Results AND Discussion}

A summary of fusion peptides originated from mtDNA deletions are given in Table 2 . Effects of D-1 deletion is outlined here as for representation. D-1 direct repeats are placed in both gene COX3 and ND5. After the deletion, the partial nucleotide sequence from both genes produced a chimeric protein that retains the upstream region (N-terminal) of COX3 and downstream region (C-terminal) of ND5 (Fig. 1 and 2). The sequence of the fusion protein was as follows:

MTHQTHAYHMVNPSPWPLTGAFSALLLTSGLVMWFHYNSITLLTLGLLTNILTMYQW WRDVIREGTYQGHHTPIVQKGLRYGMILFIVSEVFFFAGFFWAFYHSSLVPTHDLGGCW PPTGISPLNPLEVPLLNTSVLLASGVSITWAHHSLMEGKRNHMNQALLITLIATSMTAM YSMRIIYFVTMTKPRFPPLISINENDPDLMNPIKRLAFGSIFAGFVISYNIPPTSIPVLTMPW FLKTTALIISVLGFLIALELNNLTMKLSMNKANPYSSFSTLLGFFPSIIHRITPMKSLNLSLK TSLTLLDLIWLEKTIPKSTSTLHTNMTTLTTNQKGLIKLYFMSFLINIILIIILYSINLE

*Dark blue, red, and light blue color denotes COX3, common part, and ND5 gene portion respectively.

Table 2: List of the fusion proteins resulted from established and predicted deletions.

\begin{tabular}{|c|c|c|}
\hline & mtDNA Deletion & Resulting Fusion Protein (AA = amino acid) \\
\hline \multirow{6}{*}{$\begin{array}{l}\text { Proved } \\
\text { Deletions }\end{array}$} & D1 Deletion & COXIII-ND5 (165 AA of COXIII and 196 AA of ND5) \\
\hline & D3 Deletion & ND3-ND5 (37 AA of ND3 and 88 AA of ND5) \\
\hline & D13 Deletion & $\begin{array}{l}\text { No fusion protein. Truncated COXIII ( } 28 \mathrm{AA}) \text { and } \\
\text { truncated ND6(140 AA) }\end{array}$ \\
\hline & & ATP6-ND5 (19 AA of ATP6 and 107 AA of ND5) \\
\hline & D14 deletion & Or ATP6-ND5 (13 AA of ATP6 and 113 AA of ND5) \\
\hline & D17 deletion & No fusion protein. $R n r-2$ gene deletion. \\
\hline \multirow{4}{*}{$\begin{array}{l}\text { Predicted } \\
\text { Deletions }\end{array}$} & \multirow{2}{*}{ D2 deletion } & COX3-ND5 (93 AA of COX3 and $145 \mathrm{AA}$ of ND5) or \\
\hline & & $\underline{\mathrm{COX} 3-\mathrm{ND} 5}$ (97 AA of $\mathrm{COX} 3$ and $141 \mathrm{AA}$ of ND5) \\
\hline & \multirow{2}{*}{ D-16 deletion } & $\underline{\text { ND5-CYTB }}$ (49 AA of ND5 and 141 AA of CYTB) or \\
\hline & & ND5-CYTB (55 AA of ND5 and 135 AA of CYTB) \\
\hline
\end{tabular}



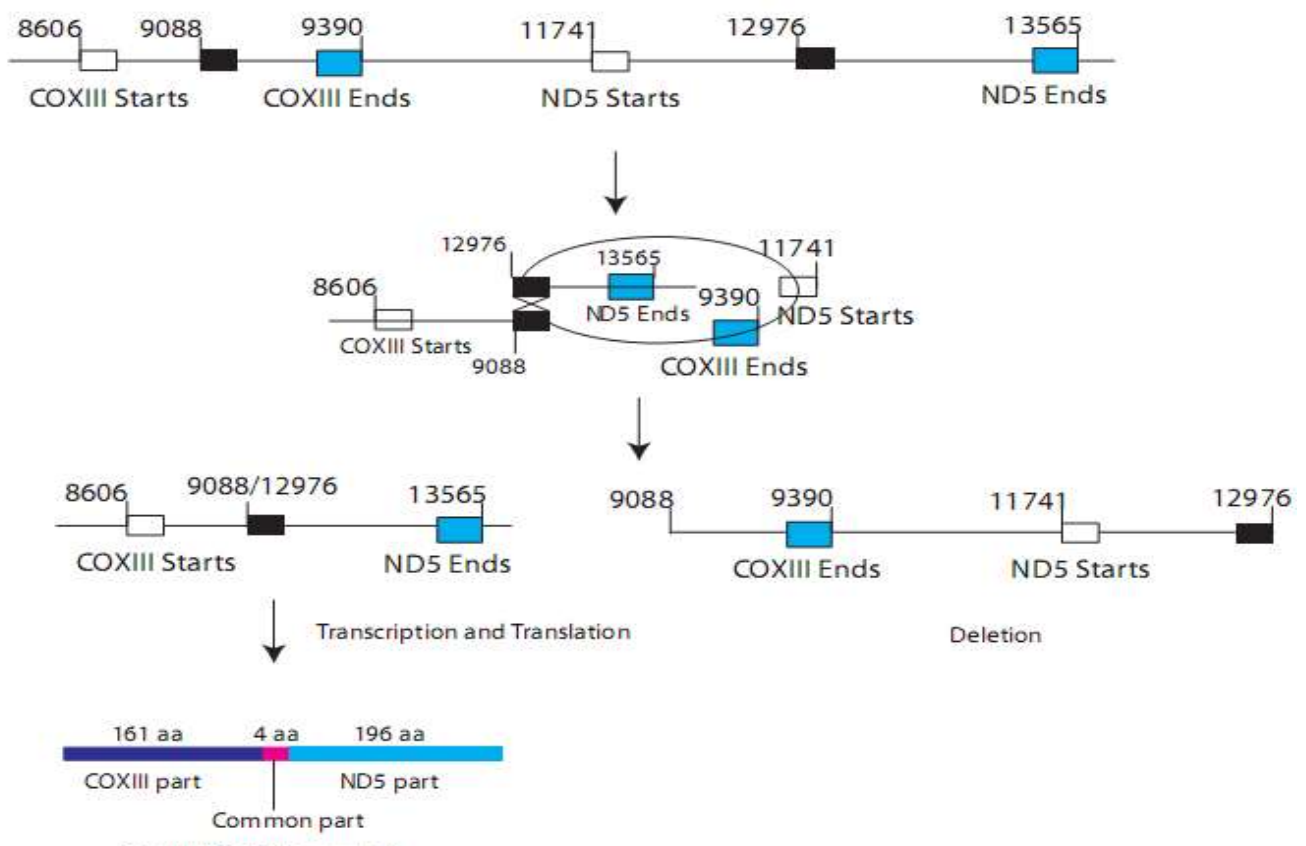

Coxlll-ND5 Fusion protein

Figure 1: Schematic representation of resulting fusion protein from D1 deletion.
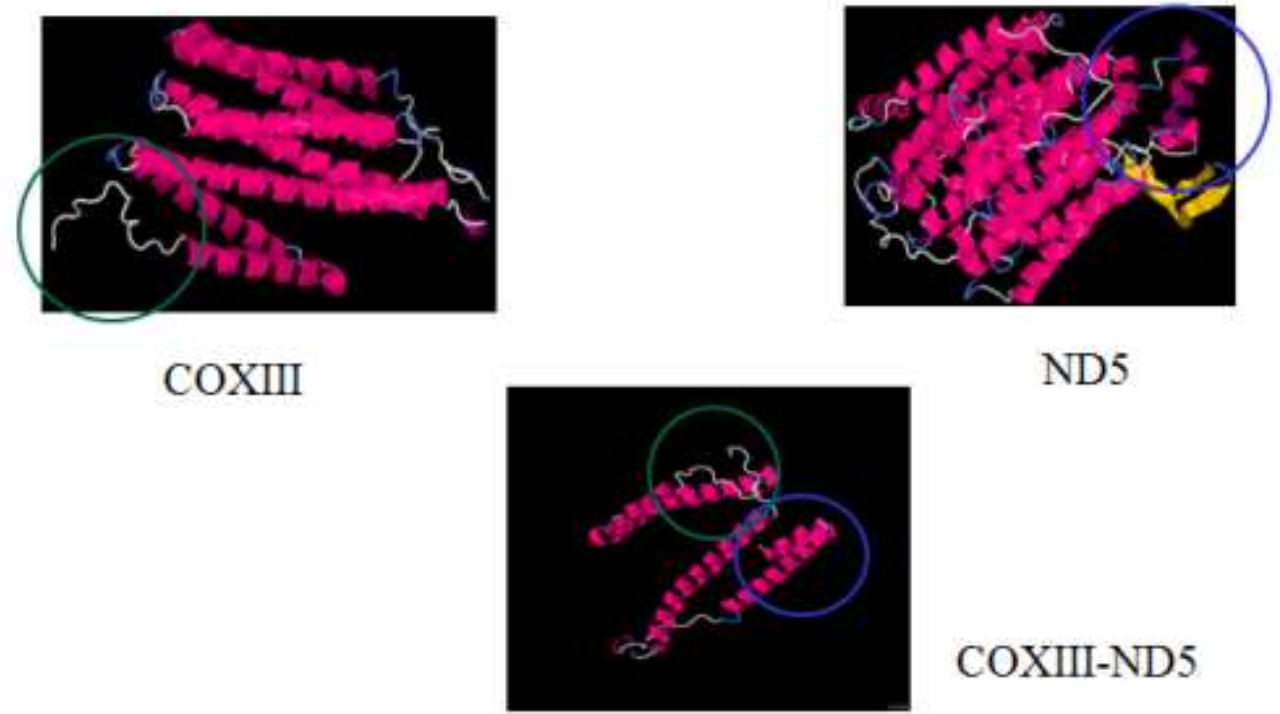

ND5

\section{COXIII-ND5}

Figure 2: Structure of full length COXIII and ND5 and the fusion protein. The fusion product retains the partial structure from the full length native structure.

From our study with correlation with others, it has been found that mtDNA deletions are excessively prominent at direct-repeat sites (Tanhauser et al., 1995). The existence of repeat may play a role in bimolecular events leading to a mtDNA deletion, but the exact mechanism is not clear and needs further investigation. However, it can be assumed that large direct repeats have the longest continuous stretches of sequence homology, they 
would have the highest frequent sites of mtDNA deletion break-points. Sequence homology was found in every direct repeat break-point in our experiment suggesting that repeats have played role not only in the production of the break-points but also in the repair of mtDNA damage (Hiraoka et al., 1998). None the less, repeats drive breakpoint generation when there is an error in mtDNA replication because of inappropriate alignment of direct repeats or a mtDNA deletion.

Recent research in mitochondrial biology demonstrated that fission and fusion have a tremendous modulatory role in mitochondrial function as diverse as apoptosis, energetics, calcium signaling, and ROS generation. Deletion in mtDNA and production of the fusion protein can induce apoptotic cell death. The question is why and how. There exists three general mechanisms those could be responsible for this phenomena. From our study, it is vivid that deletion and fusion disrupt ETS, OXPHOS, and ATP production. The release of the chimeric proteins triggers the activation of caspase family proteases and finally alteration of cellular reduction-oxidation (redox) potential. Disruption of electron transport chain has been recognized as an early feature of cell death. $\gamma$-irradiation induces mtDNA mutation and apoptosis in thymocytes and a rupture in the electron transport chain. This method is similar to ceramide-mediated disruption of ETS in cells as well as in isolated mitochondria. Fas mediated ligation also leads to a disruption in cytochrome c function in electron transport (Hiraoka et al., 1998).

Another result of the loss of electron transport could be a drop in ATP production. Drop in ATP production is also observed during apoptosis. In fact, ATP plays a vital role in downstream events in apoptosis. Although loss mitochondrial ATP production can kill a cell, it is unlikely that this is a mechanism for induction of apoptosis.

\section{ConClusion AND Significance}

The mitochondrial genome has been considered to play a critical role in aging process and development of a number of human diseases more clearly. This study illustrates the generation of fusion peptides through mtDNA deletion originated from aging and oxidative stress. Such fusion proteins may produce chimeric complexes leading to short-circuit across mitochondrial electron transport chain. These could lead to further oxidative stress and more mtDNA deletions resulting complete mitochondrial dysfunction and cell death. Hence, this study would help to understand the etiology of different mitochondrial diseases in human and their prevention more clearly.

\section{ACKNOWLEDGEMENT}

We are thankful to the Biotechnology Research Centre, University of Dhaka to fund this research.

\section{RefERENCES}

Boesch, P. et al. (2011). DNA repair in organelles: Pathways, organization, regulation, relevance in disease and aging. Biochim Biophys Acta. 1813: 186-200.

Croteau, D.L., Stierum, R.H. and Bohr, V.A. (1999). Mitochondrial DNA repair pathways. Mutat Res. 434: $137-48$.

Druzhyna, N.M., Wilson, G.L. and LeDoux, S.P. (2008). Mitochondrial DNA repair in aging and disease. Mech Ageing Dev. 129: 383-90.

Hiraoka, W., Vazquez, N., Nieves-Neira, W., Chanock, S.J. and Pommier, Y. (1998). Role of oxygen radicals generated by NADPH oxidase in apoptosis induced in human leukemia cells. J Clin Invest. 102: 1961-8 (1998). 
Jeppesen, D.K., Bohr, V.A. and Stevnsner, T. (2011). DNA repair deficiency in neurodegeneration. Prog Neurobiol. 94(2): 166-200.

Tanhauser, S.M. and Laipis, P.J. (1995). Multiple deletions are detectable in mitochondrial DNA of aging mice. J Biol Chem. 270: 24769-75.

Tann, A.W. et al. (2011). Apoptosis Induced by Persistent Single-strand Breaks in Mitochondrial Genome: critical role of ExoG in their repair. J Biol Chem 286: 31975-83.

$$
--0-
$$

